\title{
Neuroimaging of Epilepsy: Lesions, Networks, Oscillations
}

\author{
E. Abela $\cdot$ C. Rummel $\cdot$ M. Hauf $\cdot$ C. Weisstanner $\cdot$ \\ K. Schindler $\cdot$ R. Wiest
}

Received: 22 October 2013 / Accepted: 3 January 2014 / Published online: 15 January 2014

(C) Springer-Verlag Berlin Heidelberg 2014

\begin{abstract}
While analysis and interpretation of structural epileptogenic lesion is an essential task for the neuroradiologist in clinical practice, a substantial body of epilepsy research has shown that focal lesions influence brain areas beyond the epileptogenic lesion, across ensembles of functionally and anatomically connected brain areas. In this review article, we aim to provide an overview about altered network compositions in epilepsy, as measured with current advanced neuroimaging techniques to characterize the initiation and spread of epileptic activity in the brain with multimodal noninvasive imaging techniques. We focus on resting-state functional magnetic resonance imaging (MRI) and simultaneous electroencephalography/ fMRI, and oppose the findings in idiopathic generalized versus focal epilepsies. These data indicate that circumscribed epileptogenic lesions can have extended effects on many brain systems. Although epileptic seizures may involve various brain areas, seizure activity does not spread diffusely throughout the brain but propagates along specific anatomic pathways that characterize the underlying epilepsy syndrome. Such a functionally oriented approach
\end{abstract}

E. Abela, MD $(\bowtie) \cdot$ C. Rummel $\cdot$ M. Hauf $\cdot$ C. Weisstanner R. Wiest

Support Center for Advanced Neuroimaging (SCAN), University Institute of Diagnostic and Interventional Neuroradiology, Inselspital, University Hospital Bern, University of Bern, Freiburgstrasse 4, 3010 Bern, Switzerland

e-mail: eugenio.abela@insel.ch

E. Abela, MD $\cdot$ K. Schindler

Department of Neurology, Inselspital, University Hospital Bern, University of Bern, Bern, Switzerland

M. Hauf

Epilepsy Clinic Bethesda Tschugg, 3233 Tschugg, Switzerland may help to better understand a range of clinical phenomena such as the type of cognitive impairment, the development of pharmacoresistance, the propagation pathways of seizures, or the success of epilepsy surgery.

Keywords Epilepsy $\cdot$ Networks $\cdot$ Functional neuroimaging

\section{Introduction}

Epilepsy, one of the most prevalent neurological conditions [1], can be conceptually defined as the (pathological) propensity of the brain to generate seizures, i.e., clinical and electroencephalographic manifestations of paroxysmal neuronal activity [2]. The global burden of disease and the socioeconomic costs afforded by epilepsy are substantial $[1,3]$, and its consequences on cognitive, psychological, and social functioning of those who are affected are often devastating [4-6]. However, diagnosis and management of epilepsy have been refined considerably over the past two decades, and clinical structural and functional magnetic resonance imaging (MRI) made important contributions to this development [7]. Novel imaging techniques and the increasing availability of high-field MRI, together with the implementation of dedicated epilepsy protocols according to the guidelines of the International league against epilepsy, have improved the sensitivity of structural MRI, even for subtle abnormalities [8]. Overall, the largest consecutive analysis investigating 2,000 patients referred to MRI after a seizure reported abnormalities related to epilepsy in roughly $20 \%$ of the cases [9]. In patients with refractory epilepsy, structural lesions can be depicted in up to $82-86 \%$ of imaging studies by visual inspection $[10,11]$. Alternative quantitative postprocessing methods that incorporate different features of high-resolution datasets to integrate abnormalities of cor- 
tical thickness, blurring, and tissue intensity into a single composite map further enhance the sensitivity of structural MRI relative to conventional visual interpretation [12-14].

While lesion analysis and interpretation is the essential task for the neuroradiologist in clinical practice, a substantial body of epilepsy research has shown that focal lesions influence brain areas beyond the epileptogenic lesion, across ensembles of functionally and anatomically connected brain areas $[15,16]$. This has led to the hypothesis that epilepsy represents fundamentally a disease of neural networks, and that the principal task of the epileptologist should be to indentify and therapeutically modulate the core network components that generate seizures and support epilepsy, especially in pharmacoresistant cases [17]. Also, it has been argued that understanding the alterations in network structure and dynamics could improve diagnostic classification and identify new biomarkers and potential targets for therapy $[16,18]$.

In this review, we present current research on neural networksinepilepsy, gained throughresting-state functionalMRI (fMRI) and simultaneous electroencephalography(EEG)/ fMRI. We aim to show how these advanced imaging methods, beyond structural applications, can provide new insights into the neurobiology of epilepsy.

\section{The Network Concept in Epilepsy}

From a theoretical perspective, a network consists basically of two elements: discrete units, called "nodes," linked by a set of connections, or "edges" [19]. Nodes and edges can be defined across different spatial scales, e.g., neurons and axons on the smallest relevant scale or brain areas and white matter tracts on the largest scale, thus providing a conceptual framework to analyze any network in the micro- to the macroscopic domain [20]. If the connections are physical entities, for instance interconnecting white matter tracts as measured by diffusion tensor and diffusion spectrum imaging, it constitutes a network based on structural connectivity. However, edges do not need to be structural (physical) connections, but can also be functional associations, i.e., the linear or nonlinear interrelation between the temporal evolution of activity at different nodes. Such functional connectivity networks indicate, e.g., whether a set of homologous brain areas across both hemispheres show correlated activity measured as oscillations during rest or when engaged in a perceptual task. Finally, effective connectivity quantifies the influence of one node on the other [21]. This quantification can be either performed with direct electrical recordings in cellular neural networks, or inferred indirectly from time-series data, using complex biophysical models or algorithms derived from statistical physics [22, 23].
These different types of connectivity can be analyzed with neuroimaging methods (mentioned later in the text). For functional networks, multivariate methods such as independent component analysis (ICA) can be applied, using algorithms that detect spatiotemporal patterns from fMRI time-series data [24]. ICA is for the most part user independent (although choices on preprocessing steps and algorithms influence results) and has been, therefore, often used for the discovery of novel functional networks, especially in situations with weak experimental control or when no a priori hypotheses on network topography and/or activity exist. Examples of this include complex naturalistic fMRI tasks such as simulated driving $[25,26]$, or restingstate fMRI sessions in patients with brain damage [27-30]. Moreover, ICA is also a useful technique to filter out artificial patterns of structured noise in fMRI time series [31]. Another popular approach consists in defining the nodes (or "seeds") of the network a priori, extract the time series of activity from each node, and compute a matrix of interrelations, e.g., using linear or nonlinear measures [32, 33]. Units of parcellations for these matrices can be as small as a voxel [34], or encompass any number of (heuristically defined) macroscopical areas [35]; thus, the spatial resolution of the network is not only limited by the imaging sequence but also by the hypothesis of the researcher and the chosen scale of observation. However, both data-driven and hypothesisdriven functional connectivity networks are similar across studies, indicating that they indeed represent a neurobiologcally meaningful construct and not merely an artifact of methodical choices [32].

Functional networks can be characterized by a host of different mathematical measures derived from graph theory; see, e.g., [36] for a nontechnical review. Of special importance are those measures that describe integration versus segregation of function within the network, as the brain is fundamentally organized in locally specialized (segregated), but globally connected (integrated), functional modules [37, 38]. An example of an integration measure is the global efficiency [39], a metric that quantifies the ability of a functional network to transmit information. The global efficiency is the inverse of the average path length in a network; the average path length is the mean distance between all nodes [36]. A shorter between-node distance implies that information could be transmitted in a faster, more reliable way from node to node, thus increasing the efficiency of the network. In contrast, an example of a local segregation metric is the clustering coefficient, which indicates the prevalence of connected clusters in the neighborhood of an individual node [40]. A high average clustering coefficient of a node indicates that it takes part in a densely interconnected (highly clustered) neighborhood that is segregated from the rest of the network. As a consequence, locally integrated network neighborhoods might be represented by different 
neuroanatomical subsytems in the brain, e.g., motor, visual, or attentional networks. If a network is locally highly clustered, but distant nodes can still be reached via a small set of long-range connections, it is called a "small-world" network, in analogy to the sociological phenomenon that individuals in a population form close peer groups (clusters) but are still interconnected via a small set of distant acquaintances (connections) [40]. In such a small-world network, local integration is high, while the overall integrity of the network is also maintained, a configuration that seems to be optimal for biological networks like the brain [41].

\section{Resting-State fMRI and Combined EEG/fMRI}

One of the simplest ways to record functional connectivity networks, which is comparably easy to implement in clinical routine and imposes minimal requirements on neurological patients and MR personnel, is the acquisition of so-called resting-state fMRI runs of echo-planar imaging (EPI) sequences using the blood-oxygen level-dependent
(BOLD) contrast. During a resting-state fMRI experiment, a subject lies in the scanner with eyes closed without falling asleep nor performing any specific task for a short time, usually 6-20 $\min$ ([42], p. 662). In this setting, functional networks are known to show synchronized low-frequency fluctuations (typically $<0.1 \mathrm{~Hz}$ ). Covariance analysis of these fluctuations, either by ICA or seed-based correlations from neuroanatomically defined regions of interest, determines areas of the brain that form functionally connected cortico-subcortical networks, which can then be characterized with the network measures mentioned earlier in the text [43, 31]. In Fig. 1, we provide an example of such a network characterization from two small groups of patients (both $n=5$ ) with idiopathic generalized epilepsy (IGE) and mesial temporal lobe epilepsy (MTLE) with left-sided hippocampal sclerosis. In both patient groups, resting-state fMRI was acquired for 15 min with T2*-weighted EPI sequences using the BOLD contrast at $3 \mathrm{~T}$. BOLD time series were preprocessed as previously described, including motion correction, band-pass filtering, and elimination of physiological confounds [31] using the freely available software SPM8

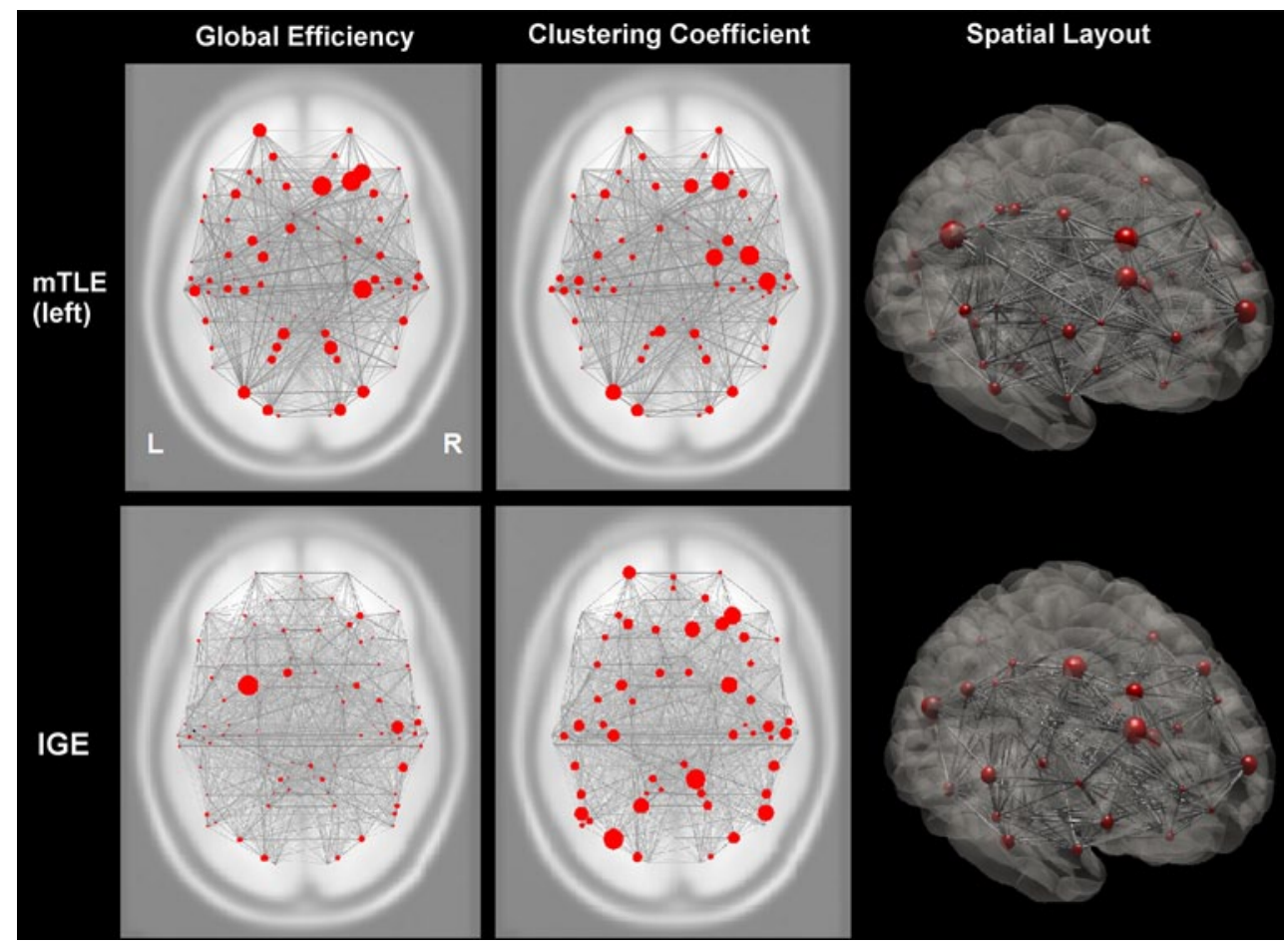

Fig. 1 Cortical functional networks in focal and generalized epilepsy syndromes. This image shows two (qualitative) applied examples of global integration and local segregation measures to cortical functional networks in mesial temporal lobe epilepsy (upper row, MTLE, $n=5$ ) and idiopathic generalized epilepsy (lower row, $I G E, n=5$ ). Red circles indicate anatomical regions (nodes); gray lines indicate the strength of interregional functional correlations (connections). The first column shows the global efficiency, which is markedly reduced over the diseased left hemisphere in MTLE and bilaterally reduced in
$I G E$, indicating syndrome-specific effects in the ability of the cortical network to transfer information. The second column shows the clustering coefficient. Clustering is reduced over the diseased hemisphere in $M T L E$, probably due to a loss of local functional modules. In contrast, clustering is more symmetrically distributed in $I G E$, suggesting that the network is fragmented in different regions. The third column illustrates the three-dimensional spatial layout of the network. Images are in neurological convention ( $L$ left, $R$ right) 
(http://www.fil.ion.ucl.ac.uk/spm/software/spm8/). A set of 90 cortical regions defined in a publicly available atlas were used as network nodes [44], and node-to-node correlations were used to define the connections in the network. We used the functional connectivity toolbox for SPM8 (http://www. nitrc.org/projects/conn/) to calculate the global efficiency and clustering coefficient of this network in both epilepsy syndromes. Both network measures indicate striking differences between syndromes: the IGE network shows a markedly reduced efficiency and increased fragmentation across both hemispheres, whereas in MTLE, only the diseased hemisphere seems to be affected. Here, however, network changes occur not only in the temporal lobe region, but also extend to fronto-parietal areas as well.

In contrast to resting-state fMRI, simultaneous EEGfMRI makes use of the complementary strengths of a neurophysiological and neuroimaging technique, i.e., the high temporal resolution of EEG and the high spatial resolution of BOLD fMRI $[45,46]$. EEG-fMRI is technically a much more demanding technique than resting-state fMRI, but has the great advantage of directly relating fMRI responses to epileptic phenomena [47-49]. Interictal discharges, which reflect the irritative zone on the EEG, are identified as intrinsic predictors to estimate the simultaneous hemodynamic responses linked to interictal epileptic discharges (IEDs). This identification is either performed by a spikebased evaluation of EEG-fMRI or by an ICA analysis of the simultaneous EEG, as recently suggested by our institution $[45,50]$. The prime justification for application of temporal ICA-based techniques for EEG decomposition holds for artifact removal, which can be regarded statistically independent from neuronal activity [51]. In practice, this technique can also be applied to isolate a single or a small number of components ("factors") containing epileptiform activity that coincides with IEDs, while the remainder does not. ICA separation on EEG recorded during MR scanning turned out insufficient but could be improved considerably after inclusion of EEG recorded outside the scanner [50]. In this way, not only single events but also the continuous dynamics of epileptiform activity can be recovered [52], and used to map the associated functional networks.

The clinical applications of combined EEG-fMRI in human epilepsy can be summarized in two categories. One concerns the investigation of BOLD correlates of epileptiform EEG signals in clinically well-defined epilepsy syndromes such as absence epilepsy (AE) or MTLE with hippocampal sclerosis. The aim of this line of investigation is not only to achieve a more complete syndrome description, but also to unravel pathophysiological mechanisms that could lead to a deeper understanding of epileptogenesis and perhaps even to a more refined, evidence-based classification of the epilepsies [2]. The second application is the localization of the epileptogenic zone during the preopera- tive workup of pediatric and adult patients suffering from pharmacoresistant epilepsies [53, 54]. Here, the focus is on localization of the seizure-onset zone, and combined EEGfMRI has proven especially valuable when no epileptogenic lesion can be identified by structural MRI using dedicated epilepsy protocols [55], or if multifocal generators are suspected [56].

\section{Networks Aspects of Idiopathic Generalized Epilepsies}

IGEs comprise a group of heterogeneous syndromes in which a strong genetic component is suspected or already identified. These syndromes have been recently reclassified as "genetic epilepsies," to avoid the ambiguous term "idiopathic" [2]. We maintain the term IGE for consistency with the fMRI literature to date. IGEs are common and account for $\sim 40 \%$ of epilepsy diagnoses. Their hallmarks are generalized spike-wave (GSW) discharges in the EEG, i.e., synchronous, mostly symmetric, bilateral paroxysmal, short ( $<200 \mathrm{~ms}$ ), high-amplitude potentials followed by a slow wave. They can occur in isolation, but classically appear in runs with frequencies of approximately 3-4 Hz [57]. Longer series of GSW discharges impair consciousness for brief periods, leading to the clinical picture of absence seizures. The latter are further characterized by sudden behavioral arrest, unresponsiveness, staring, and sometimes mild oral automatisms and/or bilateral eye blinks, and are a hallmark of $\operatorname{AE}[58,59]$.

GSW in IGE are likely generated within thalamo-cortical networks, although the relative contribution of cortical and thalamic sources remains a matter of debate [60-62]. Simultaneous EEG and fMRI were first performed with the goal to map the thalamo-cortical networks associated with GSW, but the findings of these studies have shown that the network changes are far more extended than previously thought. One of the earliest simultaneous EEG-fMRI studies by Archer et al. [63] investigated cortical and subcortical patterns of BOLD signal changes in five patients (four of them suffering from childhood AE) with frequent GSW discharges during rest. Their main finding was a large area of GSW-related BOLD deactivation in the posterior cingulate (retrosplenial cortex) that was highly consistent across patients, and more variable regions of BOLD activation in the angular gyrus and around the precentral sulcus bilaterally [63]. Thalamic BOLD activations were only seen in two of five patients and were not significant at the group level, either because of intrinsic variability of the BOLD response in subcortical structures or because of low signal-to-noise ratio at low field strengths. Subsequent studies in adults and children with IGE have reliably indentified thalamic BOLD signal increases, and have also confirmed the high degree of interindividual variability in cortical and subcorti- 


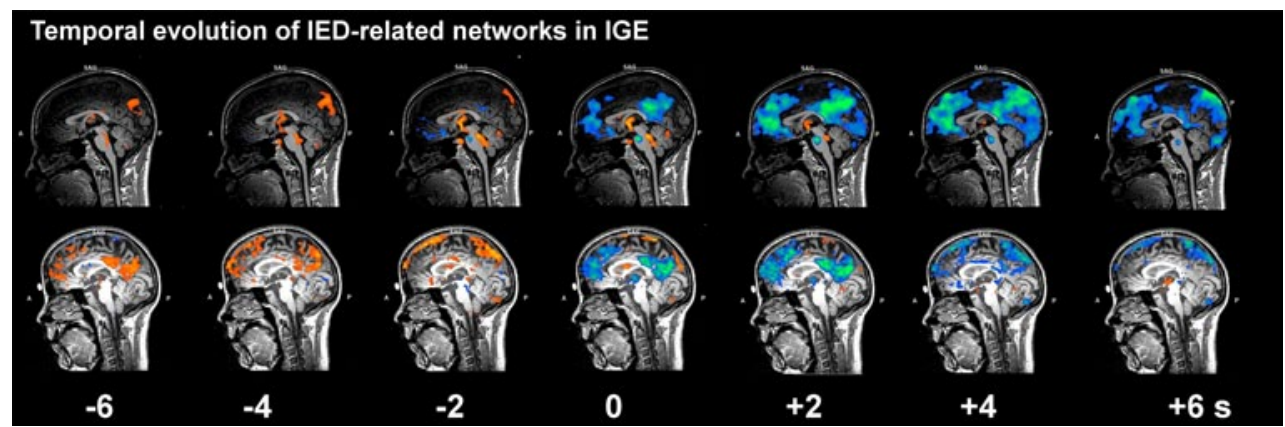

Fig. 2 Network dynamics in idiopathic generalized epilepsies. This image illustrates the temporal dynamics of networks correlated with interictal epileptiform discharges (IED) in two patients with idiopathic generalized epilepsies $(I G E)$. Zero indicates IED onset; all other numbers indicate seconds before $(-)$ or after $(+)$ the discharge. Note the

cal BOLD patterns [64-67]. The set of symmetrical regions that has been found to be commonly deactivated during GSW in IGE, i.e., precuneus, inferior parietal cortex, and ventral medial prefrontal cortex, are core nodes in the now widely known default-mode network (DMN). The DMN comprises a network that is highly active and functionally strongly interconnected during conscious rest [68, 42], and probably supports attentional and self-referential processes such as autobiographical memory or envisioning the future $[69,70]$. Although the role of this GSW-related BOLD signal changes within the DMN is still poorly understood, it is notable that deviations of DMN activity are commonly seen in neurological disorders, e.g., Alzheimer's disease or traumatic brain injury $[69,71]$.

Interestingly, recent EEG-fMRI studies have shown that activity fluctuations in the DMN and thalamus can precede EEG and behavioral changes for several seconds. The DMN, together with prefrontal regions, shows BOLD increases between 6 and $14 \mathrm{~s}$ before GSW, followed by a strong decrease during GSW that persists 7-20 s afterward $[72,73]$. The BOLD signal in the thalamus follows a different time course, with a strong increase at GSW onset (not before) that only lasts for $3 \mathrm{~s}$. Of note, interindividual variability in pre- and peri-GSW network fluctuations is high, with some cases showing either very early or very late decreases in the DMN, either short or prolonged increases in the thalamus, and different degree of overlap between activated and deactivated areas [73]. A recent study using effective connectivity modeling has found that the precuneus might have a gating or facilitatory influence on the thalamus and thus potentially on GSW generation [74]. In Fig. 2, we provide two examples of the dynamics of IED-related BOLD oscillatory activity in patients with IGE. Both were acquired with a dedicated EEG-fMRI setup at our institution as previously described $[75,76]$. The BOLD response was analyzed by first defining the onset of IED in the EEG and then shifting the hemodynamic response function in 1-s deactivation of the anterior cingulate cortex and the precuneus at time point zero in both patients, the small thalamic activation in the first patient (upper row), and the large pre-IED default mode activation in the second patient (lower row). Images courtesy of Dr. med. O. Scheidegger, Department of Neurology, Inselspital, Bern

steps from $6 \mathrm{~s}$ before $(-6)$ to $6 \mathrm{~s}$ after $(+6)$ the epileptiform activity (for a detailed description of this method, see, e.g., [72]). Early activations of cortico-subcortical networks (hot colors) and extended late deactivations of the DMN can be seen in both patients, which might account for the attentional deficits often seen in this patient group during IED.

Beyond thalamus and DMN, there is also recent evidence showing that GSWs affect a wider range of functional networks. Masterton et al. [77] have shown that also the bilateral cortical sensorimotor network (SMN) and the anterior insular network show activity changes associated with GSW. Finally, a very recent study by Zang et al. using resting-state fMRI has found that not only the aforementioned networks but also regions of cognitive and affective networks show altered patterns of functional connectivity compared with healthy controls, especially in those patients with ongoing GSW.

\section{Network Aspects of Temporal Lobe Epilepsies}

In healthy individuals, more than 20 independent networks have been identified that correspond to intrinsic and extrinsic systems, and are associated with internal- and externaloriented processing, respectively [78]. Their interaction, either between modular sensory regions to parallel-organized heteromodal processing systems or the integration between high-cognitive functional networks, is still not fully explored. There is now evidence that temporo-parietal cortices subserve as pace areas to maintain control, the default mode, and ventral attention networks [79]. The dorsal precuneus is connected to "task-positive" areas within the cognitive control network, while the ventral precuneus has stronger connections to the medial temporal lobe structures, including the hippocampus. The latter may have implications on seizure propagation in focal epilepsies, especially in the temporal lobe. 
While graph-theoretical analyses of structural and functional networks reveal a small-world organization of the cerebral cortex in healthy individuals [80], global network organization of patients reveals increased path length in temporal lobe epilepsy (TLE) [81]. Several authors demonstrated altered connectivity in patients with MTLE. As a key finding, they reported increased connections within the mesial temporal lobe (higher clustering) and decreased connectivity (longer path length) along extratemporal areas, including contralateral temporal regions [82-84]. Others suggested altered bitemporal connectivity patterns in patients with MTLE [85]. These findings suggest a deleterious impact of the epileptic lesion and the epileptogenic zone on the whole brain, potentially impacting multiple cerebral networks [86]. In contrast to TLE, only a few studies addressed functional networks associated with epilepsy syndromes with the seizure-onset zone outside the temporal lobe. One recent study compared patients with TLE versus those with frontal (FLE) and occipital lobe epilepsies [87]. The authors found that all syndromes share the deactivation of the DMN during interictal spikes, and that both FLE and TLE share an activation in the anterior insula/piriform cortex. This region has been also indentified by Laufs et al. [88] as a common node of the functional network associated with focal epilepsies. Preliminary observations of our own group revealed gray matter loss in the insula, closely related to the piriform cortex in a group of patients with neocortical structural lesions (unpublished data). Our group has furthermore examined the spatial relationship between widespread cortical atrophy and functionally connected networks linked to IED, using simultaneous EEG-fMRI in a cohort of patients suffering from MTLE [89]. We detected hemodynamic correlates to interictal epileptiform discharges beyond the seizure-onset zone in the ipsilateral insula, the temporal pole, and temporo-lateral neocortex; in the cerebellum; along the central sulcus; and bilaterally in the cingulate gyrus. We then used voxel-based morphometry, an objective measure of gray matter structure, to identify the structural network changes associated with IED [89, 90]. Widespread reductions in gray matter volume were detected in the middle and inferior temporal gyrus, the uncus to the hippocampus, the insula, the posterior cingulate, and the anterior lobe of the cerebellum. Our findings indicate a close relationship between gray matter loss and large-scale epileptic activity in MTLE. These networks encompass also the propagation pathways during seizures, as confirmed in a cohort of different patients with MTLE [91]. These results are illustrated in Fig. 3 and contrasted against results of an identical analysis

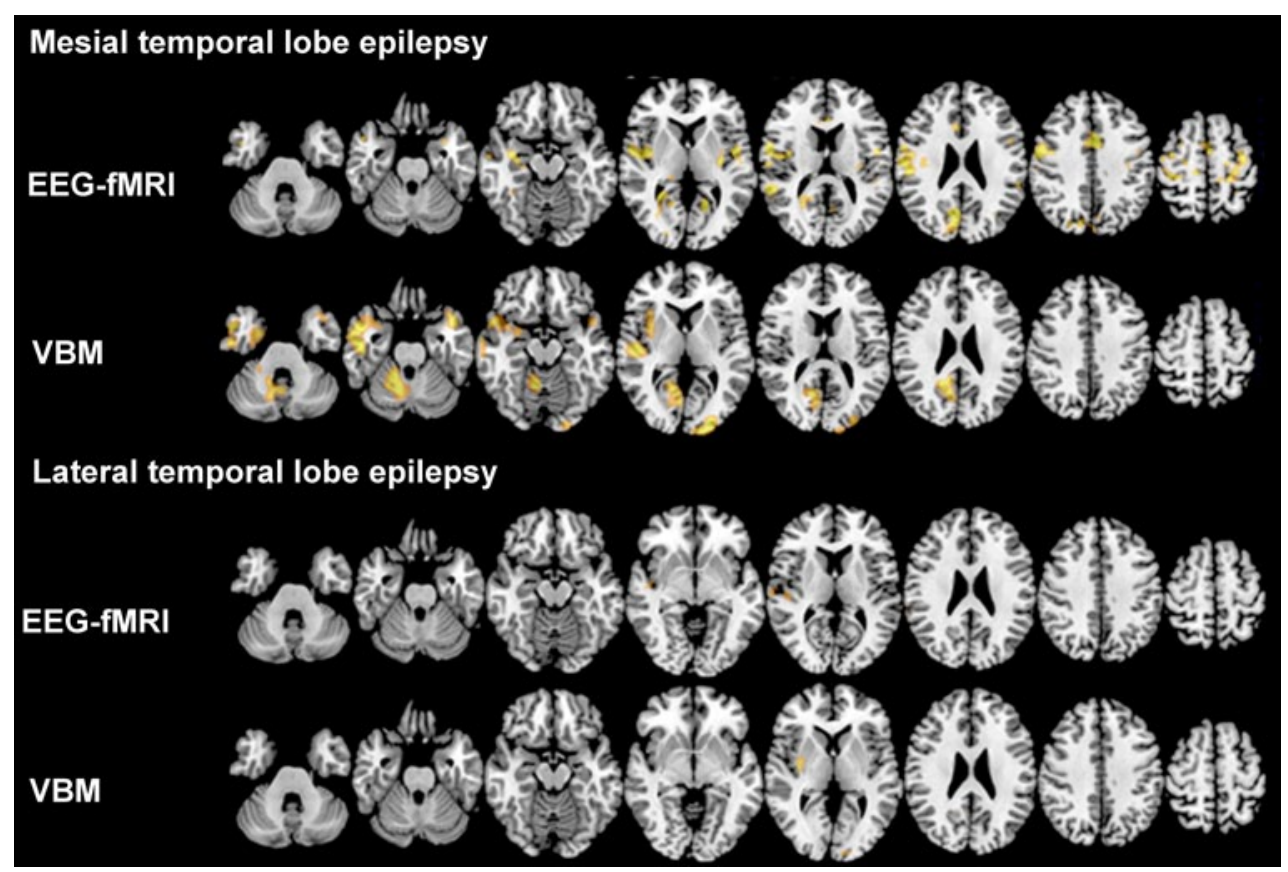

Fig. 3 Functional and structural networks in focal epilepsies. This image illustrates the concordance between functional interictal epileptic discharge (IED)-related networks derived from simultaneous electroencephalography and functional magnetic resonance imaging (EEG-fMRI) and structural networks from voxel-based morphometry $(V B M)$ in patients with mesial temporal lobe epilepsy (MTLE; $n=10$ ) versus lateral temporal lobe epilepsy (LTLE; $n=10$ ). All affected hemispheres are on the left side. Color maps indicate t-values of significant correlation with IED (EEG-fMRI) or significant gray matter volume reduction $(V B M)$. Threshold for significance was set to $t>3.1$. Despite being classified as a focal disease, widespread gray matter volume reductions can be seen in MTLE (upper panel), which overlap with the blood-oxygen level-dependent correlates to IED. This is not the case in LTLE, probably due to the heterogeneity of this syndrome. Images are in neurological convention 
in patients suffering from lateral TLE (LTLE). There are far less commonalities between functional and strucutral networks in LTLE, possibly owing to the heterogeneity of the LTLE syndrome, and also to differences in disease duration, antiseizure medication, and IED frequency, which have not been taken into account in this analysis.

Several studies that investigated alterations of gray matter volume and concentrations using MRI-based automated analysis techniques such as voxel-based morphometry and cortical thickness analysis on a whole-brain level have shown that structural changes in MTLE patients are not restricted to the hippocampus or the elements of the mesial temporal lobe [92], but show widespread abnormalities that extend into the temporal pole, temporolimbic and frontocentral regions [93, 94], and the cerebellum and the thalamus [95] and longitudinal increases in volume loss [96]. Previous ictal connectivity studies in MTLE exhibited patterns of bilateral increases of cerebral blood flow (CBF) in the temporal lobes (predominantly the middle and superior temporal gyrus, including the temporal pole, the posterior temporal lobe, and the cerebellum) and decrease CBF in the inferior temporal gyrus, the inferior parietal lobe, and the posterior cingulate [91]. Of note, there is a tight spatial overlap between hemodynamic and atrophy effects along mesolimbic areas, but not in regions beyond the limbic network [15]. Clues from morphometric analyses and combined EEG-fMRI recordings implicate that the widespread structural damage linked to abnormal hemodynamic responses is suggestive of TLE being a system rather than a focal disorder leading to a disruption of structural networks. Of note, a recent study by Voets et al. [97] investigated the three-way relationship between functional network disruption, white matter disconnection, and gray matter atrophy in patients with TLE. They found that, compared with controls, patients showed altered (typically reduced) functional connectivity between the hippocampus, anterior temporal and precentral cortices, and the default mode and SMNs. Reduced network integration of the hippocampus was explained by variations in gray matter density, while functional connectivity of the parahippocampus, and frontal and temporal neocortices, showed atypical associations with white matter coherence within pathways carrying connections of these regions. These studies again underscore the strength of the network concept to bridge not only spatial scales but also imaging modalities.

\section{Translation to Clinical Applications: Methodical Challenges}

Network analysis of functional neuroimaging data in epilepsy is a fast growing research area, but there are still many methodological issues that must be solved before this type of analysis can be used in day-to-day clinical work. While the acquisition of resting-state fMRI data is very simple and robust, any functional connectivity measures derived from such datasets are very susceptible to motion-induced artefacts. Recent studies have shown that systematic functional correlations can be induced through subject motion, leading to spurious network characteristics [98]. Appropriate measures to reduce the impact of motion artifacts and dedicated quality control is therefore of paramount importance, but there is not yet a consensus on which methods to use $[99,100]$. Also, physiological noise, i.e., respiration and pulse-related artifacts, must be accounted for, either by direct measurement $[101,102]$ or by using signals from the cerebrospinal fluid and white matter [103]. Finally, when "cleaning" and filtering resting-state fMRI data from the confounds mentioned earlier in the text, the order of processing steps must be taken into careful consideration, as noise might be reintroduced, rather than eliminated [104].

Another critical issue for clinical applications is the reliability of functional network measures and adequate benchmarks against which results of individual patient can be compared. Large-scale validation studies in healthy controls have already been undertaken, and public control fMRI databases have been set up (e.g., the "1000 Functional Connectomes Project," http://www.nitrc.org/projects/ fcon_1000/); thus, normative data are, in principle, already available. Also, there is already broad experience in the use of functional networks with other neurological diseases, in particular Alzheimer's disease, which indicates in general a good agreement between data acquired at multiple sites. However, scanner-to-scanner variability can be substantial, and there are no acquisition standards. Finally, all studies mentioned earlier in the text have used rather small sample sizes (typically <30 patients with epilepsy), which limits their generalizability. Coordinated multisite efforts are probably needed to resolve these issues.

\section{Conclusions}

We have shown, based on current evidence and on a number of illustrative examples from our current work, that neuroimaging has rapidly become a pivotal tool in the assessment of macroscopical network structure, function, and dynamics in epilepsy. Simultaneous EEG-fMRI is unique in this respect, as it can extract information on network topography and dynamics by using information from both modalities. Furthermore, recent EEG-fMRI studies have provided increasing evidence that both focal and generalized interictal epileptiform discharges are correlated with functional activation and deactivation in specific large-scale networks that span a number of cortical and subcortical nodes [16]. These data indicate that circumscribed epileptogenic lesions 
can have extended effects on many brain systems. This might help to explain a range of clinical phenomena such as the type of cognitive impairment, the development of pharmacoresistance, the propagation pathways of seizures, or the effect of resective operations [7, 105]. Thus, better understanding of altered network characteristics in epilepsy may pave a novel way for the development of biomarkers for many aspects of epilepsy and its pathophysiology [18]. Further work is needed to integrate network aspects into clinical algorithms from which patients can benefit directly.

\section{Conflict of Interest None.}

\section{References}

1. Bell GS, Sander JW. The epidemiology of epilepsy: the size of the problem. Seizure. 2002;11(Suppl A):306-14. (Quiz 15-6).

2. Berg AT, Berkovic SF, Brodie MJ, Buchhalter J, Cross JH, van Emde Boas W, et al. Revised terminology and concepts for organization of seizures and epilepsies: report of the ILAE Commission on Classification and Terminology, 2005-2009. Epilepsia. 2010;51(4):676-85. doi:10.1111/j.1528-1167.2010.02522.x.

3. Begley CE, Famulari M, Annegers JF, Lairson DR, Reynolds TF, Coan S, et al. The cost of epilepsy in the United States: an estimate from population-based clinical and survey data. Epilepsia. 2000;41(3):342-51.

4. Gaitatzis A, Sander JW. The mortality of epilepsy revisited. Epileptic Disord. 2004;6(1):3-13.

5. Gaitatzis A, Sisodiya SM, Sander JW. The somatic comorbidity of epilepsy: a weighty but often unrecognized burden. Epilepsia. 2012;53(8):1282-93. doi:10.1111/j.1528-1167.2012.03528.x.

6. Gilliam F, Hecimovic H, Sheline Y. Psychiatric comorbidity, health, and function in epilepsy. Epilepsy Behav. 2003;4(Suppl 4):S26-30. doi:S1525505003002828 [pii].

7. Richardson M. Current themes in neuroimaging of epilepsy: brain networks, dynamic phenomena, and clinical relevance. Clinical Neurophysiol. 2010;121(8):1153-75. doi:10.1016/j. clinph.2010.01.004.

8. Recommendations for neuroimaging of patients with epilepsy. Commission on Neuroimaging of the International League Against Epilepsy. Epilepsia. 1997;38(11):1255-6.

9. Craven IJ, Griffiths PD, Bhattacharyya D, Grunewald RA, Hodgson T, Connolly DJ, et al. 3.0 T MRI of 2000 consecutive patients with localisation-related epilepsy. Br J Radiol. 2012;85(1017):1236-42. doi:30177037 [pii] 10.1259/ bjr/30177037.

10. Scott CA, Fish DR, Smith SJ, Free SL, Stevens JM, Thompson PJ, et al. Presurgical evaluation of patients with epilepsy and normal MRI: role of scalp video-EEG telemetry. J Neurol Neurosurg Psychiatry. 1999;66(1):69-71.

11. Bronen RA, Fulbright RK, Spencer DD, Spencer SS, Kim JH, Lange RC, et al. Refractory epilepsy: comparison of MR imaging, CT, and histopathologic findings in 117 patients. Radiology. 1996;201(1):97-105.

12. Bernasconi A, Antel SB, Collins DL, Bernasconi N, Olivier A, Dubeau $\mathrm{F}$, et al. Texture analysis and morphological processing of magnetic resonance imaging assist detection of focal cortical dysplasia in extra-temporal partial epilepsy. Ann Neurol. 2001;49(6):770-5.
13. Besson P, Bernasconi N, Colliot O, Evans A, Bernasconi A. Surface-based texture and morphological analysis detects subtle cortical dysplasia. Med Image Comput Comput Assist Interv. 2008;11(Pt 1):645-52.

14. Wagner J, Weber B, Urbach H, Elger CE, Huppertz HJ. Morphometric MRI analysis improves detection of focal cortical dysplasia type II. Brain. 2011;134(Pt 10):2844-54. doi:awr204 [pii] 10.1093/brain/awr204.

15. Engel J Jr, Thompson PM, Stern JM, Staba RJ, Bragin A, Mody I. Connectomics and epilepsy. Current Opin Neurol. 2013;26(2):186-94. doi:10.1097/WCO.0b013e32835ee5b8.

16. Richardson MP. Large scale brain models of epilepsy: dynamics meets connectomics. J Neurol Neurosurg Psychiatry. 2012;83(12):1238-48. doi:jnnp-2011-301944 [pii] 10.1136/ jnnp-2011-301944.

17. Spencer SS. Neural networks in human epilepsy: evidence of and implications for treatment. Epilepsia. 2002;43(3):219-27.

18. Engel J Jr, Pitkanen A, Loeb JA, Dudek FE, Bertram EH 3rd, Cole AJ, et al. Epilepsy biomarkers. Epilepsia. 2013;54(Suppl 4):61-9. doi:10.1111/epi.12299.

19. Sporns O. From simple graphs to the connectome: networks in neuroimaging. NeuroImage. 2012;62(2):881-6. doi:S10538119(11)01017-2 [pii] 10.1016/j.neuroimage.2011.08.085.

20. Bullmore E, Sporns O. Complex brain networks: graph theoretical analysis of structural and functional systems. Nature Rev Neurosci. 2009;10(3):186-98. doi:nrn2575 [pii] 10.1038/ nrn2575.

21. Friston KJ. Functional and effective connectivity: a review. Brain Connect. 2011;1(1):13-36. doi:10.1089/brain.2011.0008.

22. Friston KJ, Harrison L, Penny W. Dynamic causal modelling. NeuroImage. 2003;19(4):1273-302.

23. Valdes-Sosa PA, Roebroeck A, Daunizeau J, Friston K. Effective connectivity: influence, causality and biophysical modeling. NeuroImage. 2011;58(2):339-61. doi:10.1016/j. neuroimage.2011.03.058.

24. Calhoun VD, Liu J, Adali T. A review of group ICA for fMRI data and ICA for joint inference of imaging, genetic, and ERP data. NeuroImage. 2009;45(1 Suppl):S163-72. doi:10.1016/j. neuroimage.2008.10.057.

25. Calhoun VD, Pekar JJ, McGinty VB, Adali T, Watson TD, Pearlson GD. Different activation dynamics in multiple neural systems during simulated driving. Hum Brain Mapp. 2002;16(3):158-67. doi: $10.1002 / \mathrm{hbm} .10032$.

26. Rzepecki-Smith CI, Meda SA, Calhoun VD, Stevens MC, Jafri MJ, Astur RS, et al. Disruptions in functional network connectivity during alcohol intoxicated driving. Alcohol Clin Exp Res. 2010;34(3):479-87. doi:ACER1112 [pii] 10.1111/j.1530-0277.2009.01112.x.

27. Carter AR, Astafiev SV, Lang CE, Connor LT, Rengachary J, Strube MJ, et al. Resting interhemispheric functional magnetic resonance imaging connectivity predicts performance after stroke. Ann Neurol. 2010;67(3):365-75. doi:10.1002/ana.21905.

28. Sours C, Zhuo J, Janowich J, Aarabi B, Shanmuganathan K, Gullapalli RP. Default mode network interference in mild traumatic brain injury - a pilot resting state study. Brain Res. 2013;1537:201-15. doi:S0006-8993(13)01171-2 [pii] 10.1016/j. brainres.2013.08.034.

29. Zhou Y, Milham MP, Lui YW, Miles L, Reaume J, Sodickson DK, et al. Default-mode network disruption in mild traumatic brain injury. Radiology. 2012;265(3):882-92. doi:265/3/882 [pii] 10.1148/radiol.12120748.

30. Sandrone S, Bacigaluppi M. Learning from default mode network: the predictive value of resting state in traumatic brain injury. J Neurosci. 2012;32(6):1915-7. doi:32/6/1915 [pii] 10.1523/ JNEUROSCI.5637-11.2012. 
31. Rummel C, Verma RK, Schopf V, Abela E, Hauf M, Berruecos $\mathrm{JF}$, et al. Time course based artifact identification for independent components of resting-state FMRI. Front Hum Neurosci. 2013;7:214. doi:10.3389/fnhum.2013.00214.

32. van den Heuvel MP, Hulshoff Pol HE. Exploring the brain network: a review on resting-state fMRI functional connectivity. Eur Neuropsychopharmacol. 2010;20(8):519-34. doi:S0924977X(10)00068-4 [pii] 10.1016/j.euroneuro.2010.03.008.

33. Rummel C, Abela E, Muller M, Hauf M, Scheidegger O, Wiest $\mathrm{R}$, et al. Uniform approach to linear and nonlinear interrelation patterns in multivariate time series. Phys Rev E Stat Nonlin Soft Matter Phys. 2011;83(6 Pt 2):066215.

34. Buckner RL, Sepulcre J, Talukdar T, Krienen FM, Liu H, Hedden $\mathrm{T}$, et al. Cortical hubs revealed by intrinsic functional connectivity: mapping, assessment of stability, and relation to Alzheimer's disease. J Neurosci. 2009;29(6):1860-73. doi:29/6/1860 [pii] 10.1523/JNEUROSCI.5062-08.2009.

35. Zalesky A, Fornito A, Harding IH, Cocchi L, Yucel M, Pantelis $\mathrm{C}$, et al. Whole-brain anatomical networks: does the choice of nodes matter? NeuroImage. 2010;50(3):970-83. doi:S10538119(09)01315-9 [pii] 10.1016/j.neuroimage.2009.12.027.

36. Rubinov M, Sporns O. Complex network measures of brain connectivity: uses and interpretations. NeuroImage. 2010;52(3):1059-69. doi:S1053-8119(09)01074-X [pii] 10.1016/j.neuroimage.2009.10.003.

37. Tononi G, Sporns O, Edelman GM. A measure for brain complexity: relating functional segregation and integration in the nervous system. Proc Natl Acad Sci U S A. 1994;91(11):5033-7.

38. Sporns O. Network attributes for segregation and integration in the human brain. Curr Opin Neurobiol. 2013;23(2):162-71. doi:S0959-4388(12)00189-4 [pii] 10.1016/j.conb.2012.11.015.

39. Latora V, Marchiori M. Efficient behavior of small-world networks. Phys Rev Lett. 2001;87(19):198701.

40. Watts DJ, Strogatz SH. Collective dynamics of 'small-world' networks. Nature. 1998;393(6684):440-2. doi:10.1038/30918.

41. Stam CJ, Reijneveld JC. Graph theoretical analysis of complex networks in the brain. Nonlinear Biomed Phys. 2007;1(1):3. doi:1753-4631-1-3 [pii] 10.1186/1753-4631-1-3.

42. Greicius MD, Krasnow B, Reiss AL, Menon V. Functional connectivity in the resting brain: a network analysis of the default mode hypothesis. Proc Natl Acad Sci U S A. 2003;100(1):253-8. doi:10.1073/pnas.0135058100.

43. Calhoun VD, Adali T, Pearlson GD, Pekar JJ. A method for making group inferences from functional MRI data using independent component analysis. Hum Brain Mapp. 2001;14(3):140-51. doi: $10.1002 / \mathrm{hbm} .1048$ [pii].

44. Tzourio-Mazoyer N, Landeau B, Papathanassiou D, Crivello F, Etard O, Delcroix N, et al. Automated anatomical labeling of activations in SPM using a macroscopic anatomical parcellation of the MNI MRI single-subject brain. NeuroImage. 2002;15(1):27389. doi:10.1006/nimg.2001.0978 S1053811901909784 [pii].

45. Huster RJ, Debener S, Eichele T, Herrmann CS. Methods for simultaneous EEG-fMRI: an introductory review. J Neurosci. 2012;32(18):6053-60. doi:10.1523/JNEUROSCI.0447-12.2012.

46. Ritter P, Villringer A. Simultaneous EEG-fMRI. Neurosci Biobehav Rev. 2006;30(6):823-38. doi:10.1016/j. neubiorev.2006.06.008.

47. Mulert C, Lemieux L. EEG-fMRI - phyiological basis, technique and applications. Springer; 2010. p. 309-31.

48. Moeller F, Muhle H, Wiegand G, Wolff S, Stephani U, Siniatchkin M. EEG-fMRI study of generalized spike and wave discharges without transitory cognitive impairment. Epilepsy Behav. 2010;18(3):313-6. doi:S1525-5050(10)00275-1 [pii] 10.1016/j. yebeh.2010.02.013.
49. LeVan P, Tyvaert L, Moeller F, Gotman J. Independent component analysis reveals dynamic ictal BOLD responses in EEG-fMRI data from focal epilepsy patients. NeuroImage. 2010;49(1):36678. doi:10.1016/j.neuroimage.2009.07.064.

50. Jann K, Wiest R, Hauf M, Meyer K, Boesch C, Mathis J, et al. BOLD correlates of continuously fluctuating epileptic activity isolated by independent component analysis. Neuroimage. 2008;42(2):635-48. doi:S1053-8119(08)00612-5 [pii] 10.1016/j. neuroimage.2008.05.001.

51. DelormeA, Sejnowski T, Makeig S. Enhanced detection of artifacts in EEG data using higher-order statistics and independent component analysis. NeuroImage. 2007;34(4):1443-9. doi:S10538119(06)01109-8 [pii] 10.1016/j.neuroimage.2006.11.004.

52. Onton J, Makeig S. Information-based modeling of event-related brain dynamics. Prog Brain Res. 2006;159:99-120. doi:S00796123(06)59007-7 [pii] 10.1016/S0079-6123(06)59007-7.

53. De Tiege $X$, Laufs H, Clark CA, et al. EEG-fMRI in children with pharmacoresistant focal epilepsy. Epilepsia. 2007;48(2):385-9. doi:EPI951 [pii] 10.1111/j.1528-1167.2006.00951.x.

54. Salek-Haddadi A, Diehl B, Hamandi K, Merschhemke M, Liston A, Friston K, et al. Hemodynamic correlates of epileptiform discharges: an EEG-fMRI study of 63 patients with focal epilepsy. Brain Res. 2006;1088(1):148-66. doi:S0006-8993(06)00524-5 [pii] 10.1016/j.brainres.2006.02.098.

55. Moeller F, Tyvaert L, Nguyen DK, LeVan P, Bouthillier A, Kobayashi E, et al. EEG-fMRI: adding to standard evaluations of patients with nonlesional frontal lobe epilepsy. Neurology. 2009;73(23):2023-30. doi:10.1212/WNL.0b013e3181c55d17.

56. Zijlmans M, Huiskamp G, Hersevoort M, Seppenwoolde JH, van Huffelen AC, Leijten FS. EEG-fMRI in the preoperative work-up for epilepsy surgery. Brain. 2007;130(Pt 9):2343-53. doi:10.1093/brain/awm141.

57. Weir B. The morphology of the spike-wave complex. Electroencephalogr Clin Neurophysiol. 1965;19(3):284-90.

58. Panayiotopoulos CP, Chroni E, Daskalopoulos C, Baker A, Rowlinson S, Walsh P. Typical absence seizures in adults: clinical, EEG, video-EEG findings and diagnostic/syndromic considerations. J Neurol Neurosurg Psychiatry. 1992;55(11):1002-8.

59. Holmes GL, McKeever M, Adamson M. Absence seizures in children: clinical and electroencephalographic features. Ann Neurol. 1987;21(3):268-73. doi:10.1002/ana.410210308.

60. Gloor P. Generalized cortico-reticular epilepsies. Some considerations on the pathophysiology of generalized bilaterally synchronous spike and wave discharge. Epilepsia. 1968;9(3):249-63.

61. Meeren H, van Luijtelaar G, Lopes da Silva F, Coenen A. Evolving concepts on the pathophysiology of absence seizures: the cortical focus theory. Arch Neurol. 2005;62(3):371-6. doi:62/3/371 [pii] 10.1001/archneur.62.3.371.

62. Blumenfeld H, McCormick DA. Corticothalamic inputs control the pattern of activity generated in thalamocortical networks. J Neurosci. 2000;20(13):5153-62. doi:20/13/5153 [pii].

63. Archer JS, Abbott DF, Waites AB, Jackson GD. fMRI "deactivation" of the posterior cingulate during generalized spike and wave. NeuroImage. 2003;20(4):1915-22.

64. Aghakhani Y, Bagshaw AP, Benar CG, Hawco C, Andermann $\mathrm{F}$, Dubeau F, et al. fMRI activation during spike and wave discharges in idiopathic generalized epilepsy. Brain. 2004;127(Pt 5):1127-44. doi:10.1093/brain/awh136.

65. Hamandi K, Salek-Haddadi A, Laufs H, Liston A, Friston K, Fish DR, et al. EEG-fMRI of idiopathic and secondarily generalized epilepsies. NeuroImage. 2006;31(4):1700-10. doi:10.1016/j. neuroimage.2006.02.016.

66. Moeller F, LeVan P, Muhle H, Stephani U, Dubeau F, Siniatchkin $\mathrm{M}$, et al. Absence seizures: individual patterns revealed by EEG-fMRI. Epilepsia. 2010;51(10):2000-10. doi:EPI2698 [pii] 10.1111/j.1528-1167.2010.02698.x. 
67. Moeller F, Siebner HR, Wolff S, Muhle H, Granert O, Jansen $\mathrm{O}$, et al. Simultaneous EEG-fMRI in drug-naive children with newly diagnosed absence epilepsy. Epilepsia. 2008;49(9):1510 9. doi:10.1111/j.1528-1167.2008.01626.x.

68. Raichle ME, MacLeod AM, Snyder AZ, Powers WJ, Gusnard DA, Shulman GL. A default mode of brain function. Proc Natl Acad Sci U S A. 2001;98(2):676-82. doi:10.1073/pnas.98.2.676.

69. Buckner RL, Andrews-Hanna JR, Schacter DL. The brain's default network: anatomy, function, and relevance to disease. Ann N Y Acad Sci. 2008;1124:1-38. doi:10.1196/annals.1440.011.

70. Raichle ME, Snyder AZ. A default mode of brain function: a brief history of an evolving idea. NeuroImage. 2007;37(4):1083-90. (Discussion 97-9). doi:10.1016/j.neuroimage.2007.02.041.

71. Bonnelle V, Ham TE, Leech R, Kinnunen KM, Mehta MA, Greenwood RJ, et al. Salience network integrity predicts default mode network function after traumatic brain injury. Proc Natl Acad Sci U S A. 2012;109(12):4690-5. doi:10.1073/pnas.1113455109.

72. Bai X, Vestal M, Berman R, Negishi M, Spann M, Vega C, et al. Dynamic time course of typical childhood absence seizures: EEG, behavior, and functional magnetic resonance imaging. $\mathrm{J}$ Neurosci. 2010;30(17):5884-93. doi:30/17/5884 [pii] 10.1523/ JNEUROSCI.5101-09.2010.

73. Benuzzi F, Mirandola L, Pugnaghi M, Farinelli V, Tassinari CA, Capovilla $G$, et al. Increased cortical BOLD signal anticipates generalized spike and wave discharges in adolescents and adults with idiopathic generalized epilepsies. Epilepsia. 2012;53(4):622-30. doi:10.1111/j.1528-1167.2011.03385.x.

74. Vaudano AE, Laufs H, Kiebel SJ, Carmichael DW, Hamandi K, Guye M, et al. Causal hierarchy within the thalamo-cortical network in spike and wave discharges. PloS One. 2009;4(8):e6475. doi:10.1371/journal.pone.0006475.

75. Hauf M, Jann K, Schindler K, Scheidegger O, Meyer K, Rummel $\mathrm{C}$, et al. Localizing seizure-onset zones in presurgical evaluation of drug-resistant epilepsy by electroencephalography/fMRI: effectiveness of alternative thresholding strategies. AJNR Am J Neuroradiol. 2012;33(9):1818-24. doi:10.3174/ajnr.A3052.

76. Jann K, Dierks T, Boesch C, Kottlow M, Strik W, Koenig T. BOLD correlates of EEG alpha phase-locking and the fMRI default mode network. NeuroImage. 2009;45(3):903-16.

77. Masterton RA, Carney PW, Abbott DF, Jackson GD. Absence epilepsy subnetworks revealed by event-related independent components analysis of functional magnetic resonance imaging. Epilepsia. 2013;54(5):801-8. doi:10.1111/epi.12163.

78. Doucet G, Naveau M, Petit L, Delcroix N, Zago L, Crivello F, et al. Brain activity at rest: a multiscale hierarchical functional organization. J Neurophysiol. 2011;105(6):2753-63. doi:10.1152/ jn.00895.2010.

79. Mars RB, Sallet J, Schuffelgen U, Jbabdi S, Toni I, Rushworth MF. Connectivity-based subdivisions of the human right "temporoparietal junction area": evidence for different areas participating in different cortical networks. Cereb Cortex. 2012;22(8):1894-903. doi:bhr268 [pii] 10.1093/cercor/bhr268.

80. Li ZB, Cai W, Cao Q, Chen K, Wu Z, He L, et al. (64)Cu-labeled tetrameric and octameric RGD peptides for small-animal PET of tumor alpha(v)beta(3) integrin expression. J Nucl Med. 2007;48(7):1162-71. doi:10.2967/jnumed.107.039859.

81. Bernhardt BC, Chen Z, He Y, Evans AC, Bernasconi N. Graphtheoretical analysis reveals disrupted small-world organization of cortical thickness correlation networks in temporal lobe epilepsy. Cereb Cortex. 2011;21(9):2147-57. doi:10.1093/cercor/bhq291.

82. Liao W, Zhang Z, Pan Z, Mantini D, Ding J, Duan X, et al. Altered functional connectivity and small-world in mesial temporal lobe epilepsy. PloS One. 2010;5(1):e8525. doi:10.1371/journal. pone. 0008525 .
83. Seidenberg M, Kelly KG, Parrish J, Geary E, Dow C, Rutecki $\mathrm{P}$, et al. Ipsilateral and contralateral MRI volumetric abnormalities in chronic unilateral temporal lobe epilepsy and their clinical correlates. Epilepsia. 2005;46(3):420-30. doi:10.1111/j.0013-9580.2005.27004.x.

84. Bettus G, Guedj E, Joyeux F, Confort-Gouny S, Soulier E, Laguitton $\mathrm{V}$, et al. Decreased basal fMRI functional connectivity in epileptogenic networks and contralateral compensatory mechanisms. Hum Brain Mapp. 2009;30(5):1580-91. doi:10.1002/hbm.20625.

85. Morgan VL, Rogers BP, Sonmezturk HH, Gore JC, AbouKhalil B. Cross hippocampal influence in mesial temporal lobe epilepsy measured with high temporal resolution functional magnetic resonance imaging. Epilepsia. 2011;52(9):1741-9. doi:10.1111/j.1528-1167.2011.03196.x.

86. Tracy JI, Osipowicz K, Spechler P, Sharan A, Skidmore C, Doucet $\mathrm{G}$, et al. Functional connectivity evidence of corticocortico inhibition in temporal lobe epilepsy. Hum Brain Mapp. 2014;35(1):353-66. doi:10.1002/hbm.22181.

87. Fahoum F, Lopes R, Pittau F, Dubeau F, Gotman J. Widespread epileptic networks in focal epilepsies: EEG-fMRI study. Epilepsia. 2012;53(9):1618-27. doi:10.1111/j.1528-1167.2012.03533.x.

88. Laufs H, Richardson MP, Salek-Haddadi A, Vollmar C, Duncan JS, Gale K, et al. Converging PET and fMRI evidence for a common area involved in human focal epilepsies. Neurology. 2011;77(9):904-10. doi:WNL.0b013e31822c90f2 [pii] 10.1212/ WNL.0b013e31822c90f2.

89. Wiest R, Estermann L, Scheidegger O, Rummel C, Jann K, Seeck $\mathrm{M}$, et al. Widespread grey matter changes and hemodynamic correlates to interictal epileptiform discharges in pharmacoresistant mesial temporal epilepsy. J Neurol. 2013;260(6):1601-10. doi:10.1007/s00415-013-6841-2.

90. Ashburner J, Friston KJ. Voxel-based morphometry - the methods. NeuroImage. 2000;11(6 Pt 1):805-21. doi:10.1006/nimg.20 00.0582S1053-8119(00)90582-2 [pii].

91. Weder BJ, Schindler K, Loher TJ, Wiest R, Wissmeyer M, Ritter $\mathrm{P}$, et al. Brain areas involved in medial temporal lobe seizures: a principal component analysis of ictal SPECT data. Hum Brain Mapp. 2006;27(6):520-34. doi:10.1002/hbm.20196.

92. Salmenpera T, Kalviainen R, Partanen K, Pitkanen A. Quantitative MRI volumetry of the entorhinal cortex in temporal lobe epilepsy. Seizure. 2000;9(3):208-15. doi:10.1053/seiz.1999.0373.

93. Keller SS, Roberts N. Voxel-based morphometry of temporal lobe epilepsy: an introduction and review of the literature. Epilepsia. 2008;49(5):741-57. doi:10.1111/j.1528-1167.2007.01485.x.

94. Bernhardt BC, Worsley KJ, Besson P, Concha L, Lerch JP, Evans AC, et al. Mapping limbic network organization in temporal lobe epilepsy using morphometric correlations: insights on the relation between mesiotemporal connectivity and cortical atrophy. NeuroImage. 2008;42(2):515-24. doi:10.1016/j. neuroimage.2008.04.261.

95. Riederer F, Lanzenberger R, Kaya M, Prayer D, Serles W, Baumgartner C. Network atrophy in temporal lobe epilepsy: a voxel-based morphometry study. Neurology. 2008;71(6):419-25. doi:10.1212/01.wnl.0000324264.96100.e0.

96. Cascino GD. Temporal lobe epilepsy is a progressive neurologic disorder: time means neurons! Neurology. 2009;72(20):1718-9. doi:10.1212/WNL.0b013e3181a4e465.

97. Voets NL, Beckmann CF, Cole DM, Hong S, Bernasconi A, Bernasconi N. Structural substrates for resting network disruption in temporal lobe epilepsy. Brain. 2012;135(Pt 8):2350-7. doi:10.1093/brain/aws137.

98. Power JD, Barnes KA, Snyder AZ, Schlaggar BL, Petersen SE. Spurious but systematic correlations in functional connectivity MRI networks arise from subject motion. NeuroImage. 2012;59(3):2142-54. doi:S1053-8119(11)01181-5 [pii] 10.1016/j.neuroimage.2011.10.018. 
99. Power JD, Mitra A, Laumann TO, Snyder AZ, Schlaggar $\mathrm{BL}$, Petersen SE. Methods to detect, characterize, and remove motion artifact in resting state fMRI. NeuroImage. 2013;84C:320-41. doi:S1053-8119(13)00911-7 [pii] 10.1016/j. neuroimage.2013.08.048.

100. Power JD, Barnes KA, Snyder AZ, Schlaggar BL, Petersen SE. Steps toward optimizing motion artifact removal in functional connectivity MRI; a reply to Carp. NeuroImage. 2013;76:439-41. doi:S1053-8119(12)00290-X [pii] 10.1016/j. neuroimage.2012.03.017.

101. Tijssen RH, Jenkinson M, Brooks JC, Jezzard P, Miller KL. Optimizing RetroICor and RetroKCor corrections for multi-shot 3D FMRI acquisitions. NeuroImage. 2013;84C:394-405. doi:S10538119(13)00926-9 [pii] 10.1016/j.neuroimage.2013.08.062.

102. Glover GH, Li TQ, Ress D. Image-based method for retrospective correction of physiological motion effects in fMRI: RETROICOR. Magn Reson Med. 2000;44(1):162-7. doi:10.1002/15222594(200007)44:1<162::AID-MRM23>3.0.CO;2-E [pii].
103. Behzadi Y, Restom K, Liau J, Liu TT. A component based noise correction method (CompCor) for BOLD and perfusion based fMRI. NeuroImage. 2007;37(1):90-101. doi:S10538119(07)00383-7 [pii] 10.1016/j.neuroimage.2007.04.042.

104. Hallquist MN, Hwang K, Luna B. The nuisance of nuisance regression: spectral misspecification in a common approach to resting-state fMRI preprocessing reintroduces noise and obscures functional connectivity. NeuroImage. 2013;82:208 25. doi:S1053-8119(13)00626-5 [pii] 10.1016/j. neuroimage.2013.05.116.

105. Murta T, Leal A, Garrido MI, Figueiredo P. Dynamic Causal Modelling of epileptic seizure propagation pathways: a combined EEG-fMRI study. NeuroImage. 2012;62(3):1634-42. doi:S10538119(12)00541-1 [pii] 10.1016/j.neuroimage.2012.05.053. 